\title{
A Fuzzy-Logic Advisory System for Lean Manufacturing within SMEs
}

Pius Achanga, Essam Shehab, Rajkumar Roy, Geoff Nelder \{p.achanga; e.shehab; r.roy\}@ cranfield.ac.uk; gnelder@gnelder.freeserve.co.uk Manufacturing Department, Cranfield University, Cranfield, Bedford MK43 0AL

\begin{abstract}
This research paper presents the development of a fuzzy-logic advisory system to assist smallmedium size companies (SMEs) as a decision support tool for implementing lean manufacturing. The system is developed using fuzzy logic rules, with a combination of research methodology approaches employed in the research study that included data collection from ten manufacturing SMEs through documentation analysis, observation of companies' practices and semi-structured interviews. The overall system comprises three fuzzy-logic advisory sub-systems that feed into a main system. These outputs are relative cost of lean implementation, a company lean readiness status and the level of value-add to be achieved (impact/benefits). The three sub-systems were validated with hard data that enabled the assignment of a number of input variables whose membership functions aided the definition of the linguistic variables used. The main system yielded heuristic rules that enable the postulation of scenarios of lean implementation (Do-it, Probably doit, Possibly do-it and Do not do-it). This was also validated with a number of firms based within the UK. Moreover, expert opinions encompassed those in both academic and industrial settings.
\end{abstract}

The developed system has the capability to assess the impact of implementing lean manufacturing within small-to-medium sized manufacturers. Hence, a major contribution of the developed system is its provision of the heuristic rules that aid decision-making process for lean implementation at the early implementation stage. The visualisation facility of the developed system is also a useful tool in enabling potential lean users to forecast the relative cost of the lean project upfront, anticipate lean benefits, and realise the degree of lean readiness.

Key words: Fuzzy logic; Impact assessment; Lean manufacturing; SMEs 


\section{Introduction}

Traditional cost management systems are deemed ineffective in measuring the relative cost and the likely value-add returns on any prospective lean manufacturing project (Northrup, 2005). Few companies are able to assess the impact of lean manufacturing at an early stage, in order to determine its viability. This stalemate creates an atmosphere of ambiguity amongst potential UK small-to-medium size manufacturing (SMEs) lean users (Achanga et al. 2006a), and adds to the challenges of implementing lean manufacturing within the UK SMEs. Moreover, the survivability of most UK SMEs is challenged by the heightened market competitiveness brought about by factors such as globalisation and emerging technologies. These in turn impact greatly on their performance strategy, since cost analysis is regarded as one of the most critical performance strategies in any business undertaking (Agyapong-Kodua et al.2009, Roy, 2003). The use of lean manufacturing as a productivity improvement initiative that eliminates waste within manufacturing environments, consequently reducing the cost of manufacture, is proposed as an ideal solution. This is worthy of attention because SMEs play a pivotal role in the UK Government strategies for economic development (Achanga, 2007; Denton, 1997; Levy, 1993). The research set out in this paper aims to address the problem of SMEs inability to embrace lean manufacturing practices by developing a fuzzy-logic advisory system capable of aiding SMEs practitioners in the decision-making process on whether to implement lean manufacturing or not. This idea is derived from the fact that fuzzylogic uses knowledge about a specific domain to arrive at a solution to a problem as demonstrated by authors such as (Rao and Pratihar, 2006; Parent et al.2007; Lau et al.2005; Muthus et al.2001). The research project identifies factors that determine the assessment of lean manufacturing. Moreover, the developed system has been validated through industrial expert opinions.

The remainder of the paper is structured in five sections. Section 2 highlights related work in the area of fuzzy logic system development and application. Section 3 describes the research methodology adopted and the data collection process used. In section 4 a presentation and discussion of the system development phases is conducted. The validation process of the developed system is demonstrated in section 5; while Section 6 discusses and concludes the overall research work presented. 


\section{Related Research}

The application of most productivity improvement initiatives such as lean manufacturing within large size enterprise has been extensive (Hines et al.2004; Shah and Ward, 2003; Cook and Graser, 2002; Murman et al.2002; Panizzolo, 1998, Reid and Koljonen, 1999). Conversely, the issue of best practice adoption within SMEs has always posed many practical, theoretical, financial and organisational challenges (Stanworth and Purdy, 2003). There appear various reasons pertaining to this stalemate. The cost of implementing new ideas and a lack of understanding and the misapplication of tools and techniques are some of the pertinent issues documented by scholars such as Hicks and Mathews (2010; Zulfiqar, 2007). Moreover, most SMEs practitioners are not able to perform the likely cost-benefit analysis of implementing productivity improvement initiatives such as lean manufacturing. This is particularly difficult due to the lack of precise data to realise such objectives.

The use of fuzzy logic has been adopted in this research project as a means of resolving the problem of data imprecision. This is because several researchers have adopted the use of fuzzy logic in achieving desirable outcomes with limited or incomplete data. This involvement is claimed because the approximate reasoning of a fuzzy set theory can properly represent linguistic terms (Shehab and Abdalla, 2002). Moreover, fuzzy logic (FL) in the sense of the fuzzy set (FS) theory was invented in the mid-sixties as a mathematical framework for formalising the theory of approximate reasoning (Liang and Wang, 1991). The proponents of fuzzy systems have argued that a fuzzy set theory has the capability of capturing the uncertainty under conditions of incomplete, non-obtainable and unquantifiable information (Kulak et al. 2005). Hence, a fuzzy logic model for assessing the quality of steel production was also developed (Collantes et al.1999). Their inference stems from the fact that a fuzzy logic approach handles missing data for decision-making. Therefore, fuzzy logic in this instance provided the possibility of filling the incomplete records by looking for different trends and patterns in the database.

A hierarchy related to the decision problem for selecting value stream mapping tools in a lean manufacturing context was also developed (Singh et al. 2006). The previous authors' efforts evolved from the problems associated with the complexity in selection of detailed mapping tools for the identification of waste at a micro-level. The deployment of a fuzzy approach mapped the linguistic relationships that exist between the wastes and the tools to drive out the imprecision and 
vagueness in the relationships, hence providing a significant tool for decision-making. Never-theless, contemporary research contributions as discussed above, have not pursued the potential of fuzzy expert system deployment as a mechanism of assessing lean impact at the early implementation stage (Achanga et al. 2006b). There does not appear significant contribution towards fuzzy logic system adoption in determining cost-benefit analysis within lean manufacturing. Hence, a fuzzy logic system for lean implementation within SMEs was proposed (Achanga, 2007).

\section{Data Collection and Analysis}

The research work set out in this paper adopted qualitative research technique in its investigation. This encompassed company visits, documentation analysis and interview techniques as preferred methods of data collection in determining the current practice of lean implementation within these companies, as illustrated in Figure (1). The objective of determining the current practice was to explore the operational activities of the investigated SMEs, so as to determine their major business drivers.

The industrial research study was also intended to investigate how SMEs currently practice lean manufacturing concepts and then formulate the current status (AS-IS model), as a means of solving the existing research gaps. Moreover, the data collection process also involved an investigation of how lean manufacturing specialists deploy lean concepts within SMEs. The investigation adopted a questionnaire design as a means of enabling the study to obtain meaningful outcomes from this research project. The questionnaire was developed to include the following areas:

- Type of product(s) a particular SME manufactures and its volume level classified herein as (high, medium and low);

- Companies' size in terms of employees and annual turnover;

- Management style (whether independent or owner-managed), and structure of the organisations;

- Companies' major business drivers and the manufacturing issues;

- The strengths and weaknesses of the organisations, including their potential sources of competitive advantage and finally;

- The current status of their lean manufacturing applications. 
These companies were engaged consistently in terms of information gathering for the entire research project life. Some of the questions that aided the identification of the research focus were as follows:

- Is this company independently managed?

- What are the major drivers of your business?

- What is your definition of lean manufacturing?

- What has motivated the company to implement lean manufacturing?

- To what extent has lean manufacturing been implemented in your organisation (piecemeal or wholesome)?

- What were the criteria for choosing that specific area?

- How many people were involved in the exercise?

- What training if any, did the staff undertake?

- What were the difficulties encountered in training and how were they overcome?

- What were the direct and indirect costs (e.g. labour costs and consultancy fees), involved in the implementation of lean manufacturing?

The study thus embraced a number of significant characteristics within the investigated companies. The aim was to obtain first-hand information on their manufacturing issues. The characteristics of the visited companies are presented in Table (1). Relevant data such as lead-time, delivery-time and profit figures were also captured. This information was retrieved through the observation of activities, and the interviewing of key experts to bolster the assessment made on the observation exercises. Moreover, the retrieved data were analysed by a comparative assessment of what these companies reported and the literature review findings. A verification exercise was done through expert opinion as a means of validating the findings. 


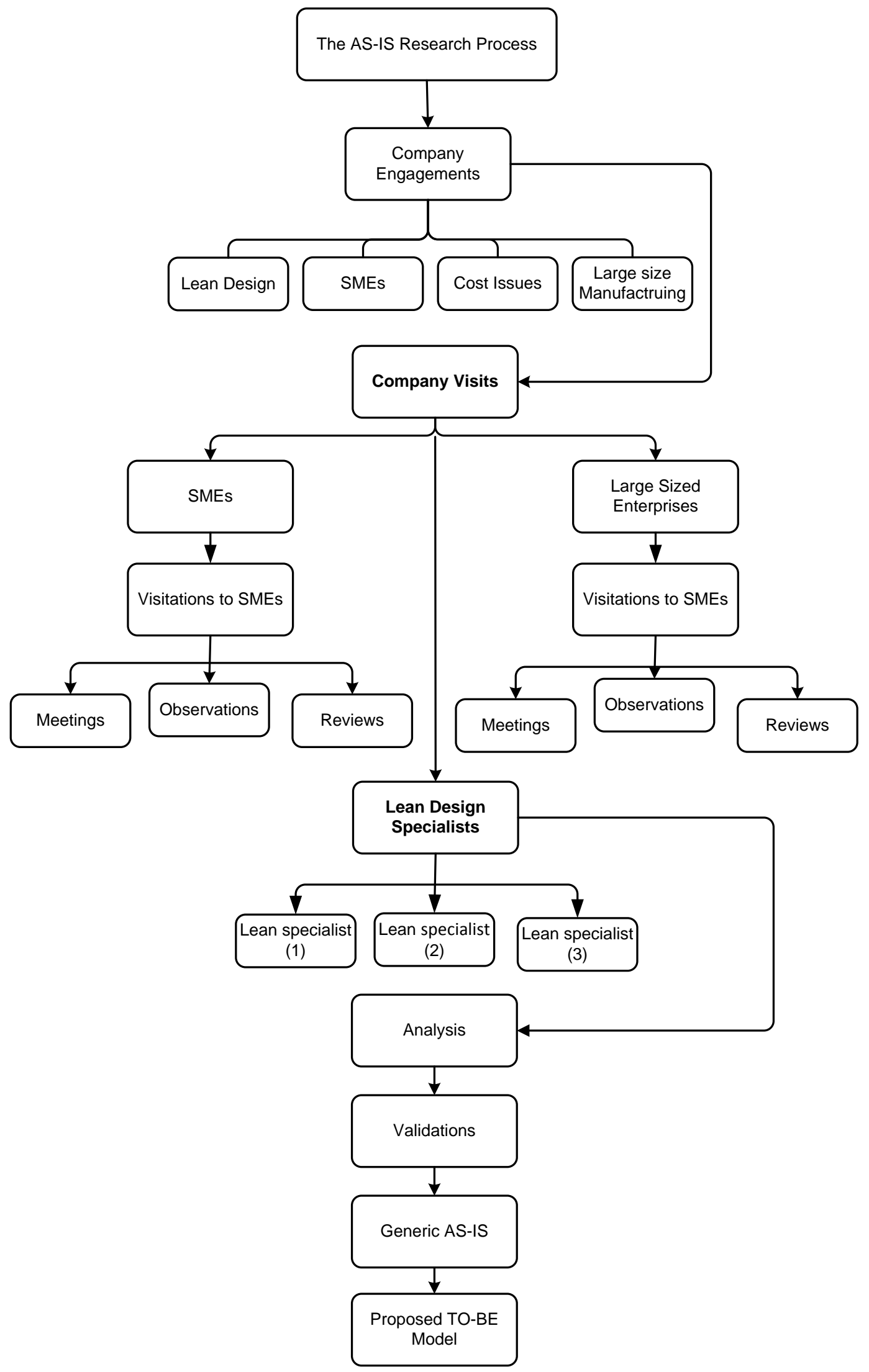

Figure 1: Industrial data collection process 
Table 1: Characteristics of the investigated SMEs

\begin{tabular}{|l|c|c|c|c|c|c|c|c|c|c|}
\hline \multirow{2}{*}{ Characteristics } & \multicolumn{7}{|c|}{ Investigated SMEs } \\
\cline { 2 - 14 } & 1 & 2 & 3 & 4 & 5 & 6 & 7 & 8 & 9 & 10 \\
\hline Management type & IM & IM & OM & IM & IM & OM & IM & IM & IM & IM \\
\hline Annual turnover (£) millions & 3.50 & 4.00 & 0.75 & 5.00 & 3.50 & 2.00 & 2.10 & 4.00 & 5.85 & 1.00 \\
\hline Volume of production & L-H & L-H & L & L-H & M & L-H & M & M & H & M \\
\hline Area lean applied & P & P & W & P & P & P & P & P & P & P \\
\hline Duration of lean implementation (days) & 10 & 15 & 10 & 10 & 18 & 12 & 10 & 10 & 15 & 10 \\
\hline No. of employees & 65 & 98 & 15 & 65 & 200 & 9 & 36 & 25 & 80 & 30 \\
\hline No. of employees involved & 13 & 50 & 12 & 20 & 25 & 5 & 10 & 8 & 15 & 12 \\
\hline Total spend (f) thousands & 5.0 & 4.0 & 2.5 & 10.0 & 4.0 & 2.0 & 2.7 & 1.5 & 3.5 & 1.5 \\
\hline Return on investment (ROI) (£) & 0.12 & 0.50 & 0.05 & 0.40 & 0.55 & 0.01 & 0.30 & 0.17 & 0.25 & 0.19 \\
\hline Reduction in lead times (weeks) & $6-2$ & $4-2$ & $8-3$ & $4-2$ & $6-2$ & $5-2$ & $6-3$ & $6-2$ & $6-2$ & $4-2$ \\
\hline
\end{tabular}

Key: $\mathrm{IM}=$ independently managed, $\mathrm{OM}=$ owner managed, $\mathrm{L}=$ low, $\mathrm{M}=$ medium, $\mathrm{H}=$ high, $\mathrm{P}=$ piecemeal, $\mathrm{W}=$ whole

\section{System Development Process}

The system development process passed through three stages. Firstly the data collected from companies who had previously implemented lean manufacturing were analysed. In particular, a number of lean impact factors were identified and critically analysed. This activity entailed the involvement of a number of parameters namely: cost of lean implementation, a company lean readiness status and the value-add returns (benefit/impact). The second stage of the system development process involved the design of three fuzzy-logic advisory sub-systems and the main fuzzy-logic system, as illustrated in Figure (2). Finally the developed systems were validated with a number of firms based within the UK as detailed in (Achanga, 2007). 


\subsection{Development of the Fuzzy-Logic Advisory Systems}

The first activity in the system development process was the identification of the necessary input and output parameters for the envisaged fuzzy-logic advisory system. However, preceding this phase, there was the analytical assessment of the probable parameters involved in lean impact assessment. Information for this task was derived from two distinct lean parameters; cost and Benefit/Impact of lean implementation. The cost parameters are subdivided into direct and indirect costs. Direct costs are those that are measurable and easier to identify and calculate; such as cost of hiring a consultant and training fees. However, indirect costs are difficult to identify let alone measure. They are costs such as cost of work-in-progress as a result of lean implementation halting productivity.

Moreover, on completion of the lean implementation, a company anticipates desirable outcomes as benefits/impact to its investments. This Benefit/Impact, is both tangible and intangible. Tangible benefits are those that can easily be measured such as reduction of lead-time, lower inventory levels and increased productivity. Intangible benefits are those that can be hard to identify let alone measure. Examples of these can be the level of employees' motivation and positive attitude towards change. Thus, it was realised that the defined lean input and output parameters illustrated in Figure 2, needed some extra parameters in order to consolidate a concrete and robust system. Hence, a third input parameter referred to as lean readiness was selected to achieve this objective.

To indicate the findings, the researchers thus presented the above analytical assessment to a number of practitioners within the companies highlighted in Table (1), for the expert view purposes. Specifically, they were asked whether the presented inputs (cost and readiness of lean), and output parameters (Benefit/Impact of lean), in their opinion provide realistic and relevant factors in conducting lean impact assessment. These exercises were subjective in that the researchers needed to formulate a meaningful and an acceptable system within the SME community. It is important to reiterate that the dataset used at this point, was company reported and validated in a number of workshops and case studies.

Satisfactory confirmation thus led to the design of three separate systems, based on each parameter identified in the analytical assessment. These systems were later named as sub-system 1, 2, and 3, as demonstrated in Figure (2) and had the following details. In sub-system 1, the input parameters 
were company size, lean readiness and lean impact. The output parameter in sub-system 1 was Relative Cost of lean implementation. The rationale behind adopting these parameters (input and output), was to aid decision-making on what would be the relative cost of lean implementation based on the selected input parameters (company size, lean readiness and lean impact). For example, one would be able to understand the relative cost of lean implementation if he/she knew the size of the company, and that company's lean experience in terms of the employees educational status, and the level to which the company requires lean to be applied (piecemeal or wholesale).

In terms of sub-system 2, the selected input parameters are management support, financial strength, corporate strategy and employee educational level. This system has an output parameter of Lean Readiness. These parameters were selected because it was believed a company's lean readiness and its type of management system (leadership style), in conjunction with its company culture are critical. Additionally, implementing lean manufacturing within a business attracts financial commitments. Therefore, the stronger the level of a company's finances, might imply that Such a company well positioned to release funds towards the lean cause.

The third sub-system contained the following selected input parameters: InventoryLevel, ManufacturingProcess, MotivationLevel and LeadTime. In this instance, the output parameters were defined as Lean Impact. This implies that a company that wishes to implement lean expects to achieve high impact (benefits) which are measurable in terms of low level inventories, simple manufacturing processes, high employee motivational levels and short lead-times.

These three sub-systems were developed using fuzzy logic rules because of their capability to handle imprecise data that can be translated for decision-making purposes. Each of the sub-systems generated a number of heuristic rules. In the case of sub-system 1, 20 heuristic rules were realised. Sub-system 2 and 3 generated 29 and 36 heuristic rules respectively, as exemplified in Rules (1-3), respectively. When the three sub-systems were ready; they were also validated by experts. Most of them had been involved in the validation of the data used in the system. Expert opinion were sought to verify the system's relevance, accuracy and usability. It was realised the outputs from the three sub-systems could be better utilised in a main system. 


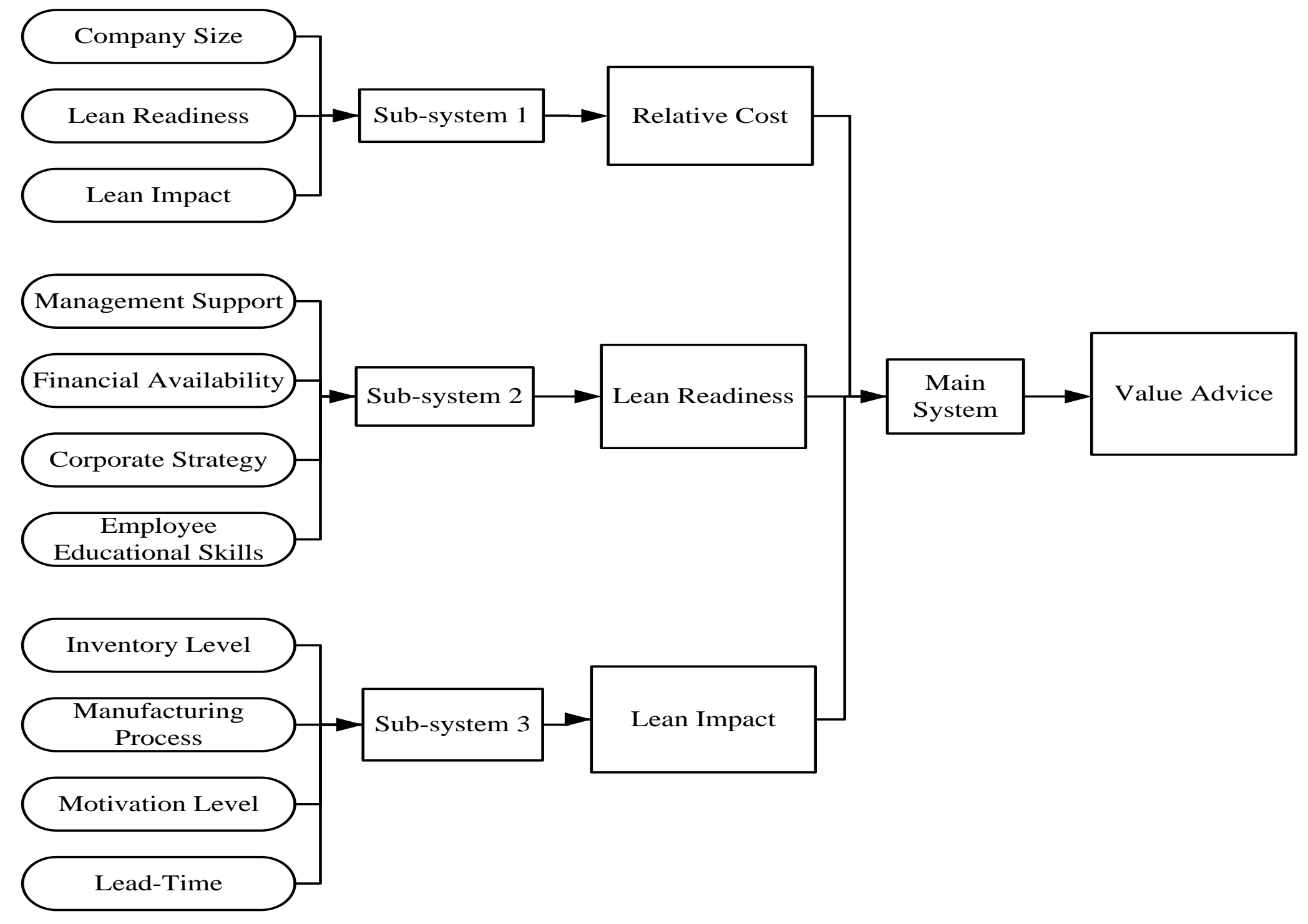

Figure 2: Architecture of the overall structure of the developed system 
Rule 1: An example of heuristic rule in sub-system 1

If compnysize is small

And companylean experience is FisrtTtimeuser

And LeanImpact area is Awareness

Then cost is VerySmall

Rule 2: An example of heuristic rule in sub-system 2

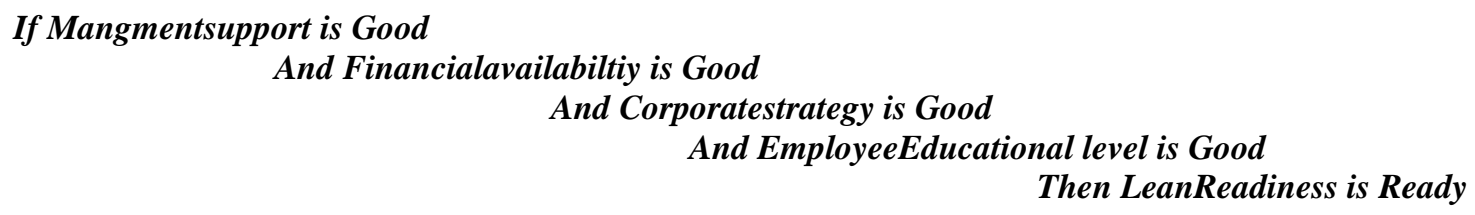

Rule: 3: An example of heuristic rule in sub-system 3

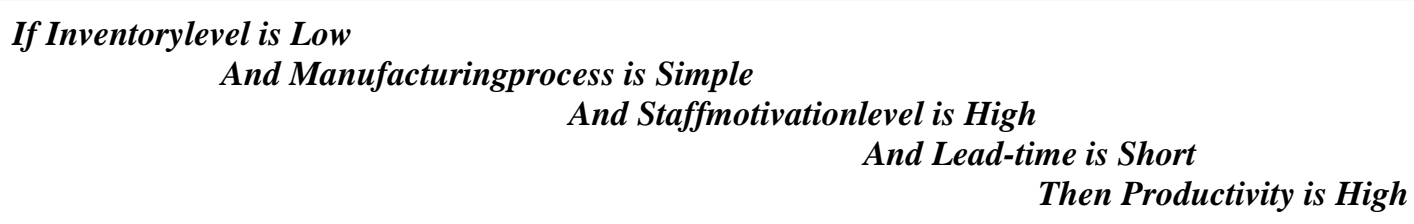

Consequently, a main system was designed using the following input parameters, Relative Cost, Lean Readiness, and Lean Impact. The defined output parameter became Value Advice. However, the input parameters used in the main system were obtained from the outputs of the three sub-systems. A number of heuristic rules were then generated using different combinations.

This process was extensive, since the visualisation facility in the developed system did not provide adequate reading at such points in time. The researchers therefore sought different expert opinions both in academia and industry as a measure of achieving better results. Eventually, eight heuristic rules were generated as the final set, and whose details are exemplified in Rule (4). These rules were also validated by experts at different levels. For example, the experts were provided with the membership functions 
and were allowed to make combinations of the linguistic variables to create a single rule of their choice. The topography of the screen viewer was analysed and adjustments made on both the rule viewer and the membership function curves. This process involved a number of experts, where they concurred with the final eight rules.

\subsection{System Functionalities}

The developed fuzzy-logic advisory system presented in this research paper performs a number of functions. These include; a demonstration of the lean implementation factors such as the relative cost of lean implementation. The system also highlights a company lean readiness status and the eventual value-add expectations thereafter. These functionalities are aided by the developed system's interfaces demonstrated in Figures (3-10) respectively. First, the system interfaces enable a user to create the fuzzy inference system (FIS) of the value advice system (see Figure 3) as a whole.

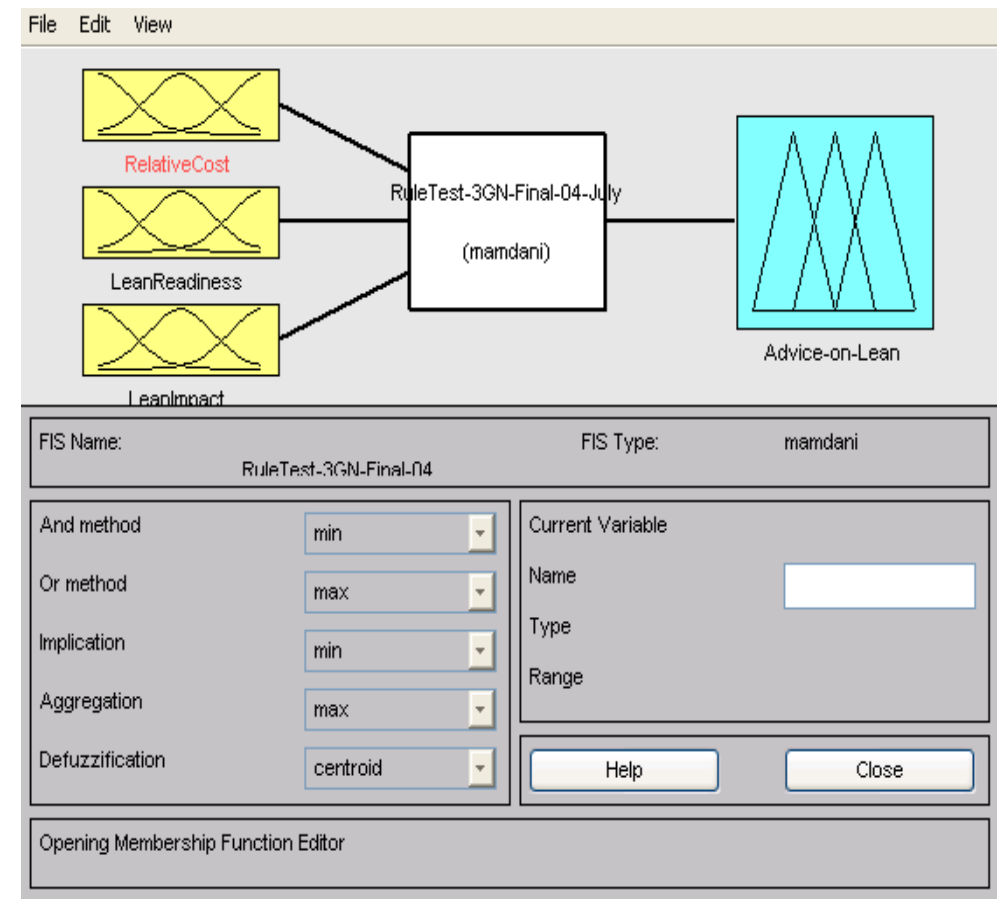

Figure 3: Interface highlighting FIS of the final system

The FIS are built with the help of the highlighted parameters: relative cost, lean readiness and lean impact. These factors can be created with the facilitation of the membership functions that provide a user with the following options: (1) Defining the linguistic variables, (2) moderating the membership functions to enable the system to realise realistic results. Figure (4) exemplifies a scenario of the relative cost 
membership functions formation. As evident from this figure, the FIS have the following parameters: company size, lean readiness and lean impact, and depended on the expected outcomes. For example, the membership function of the cost parameters was defined using linguistic variables such as: VerySmall, Small, Large and VeryLarge. These linguistic variables represent numerical values.

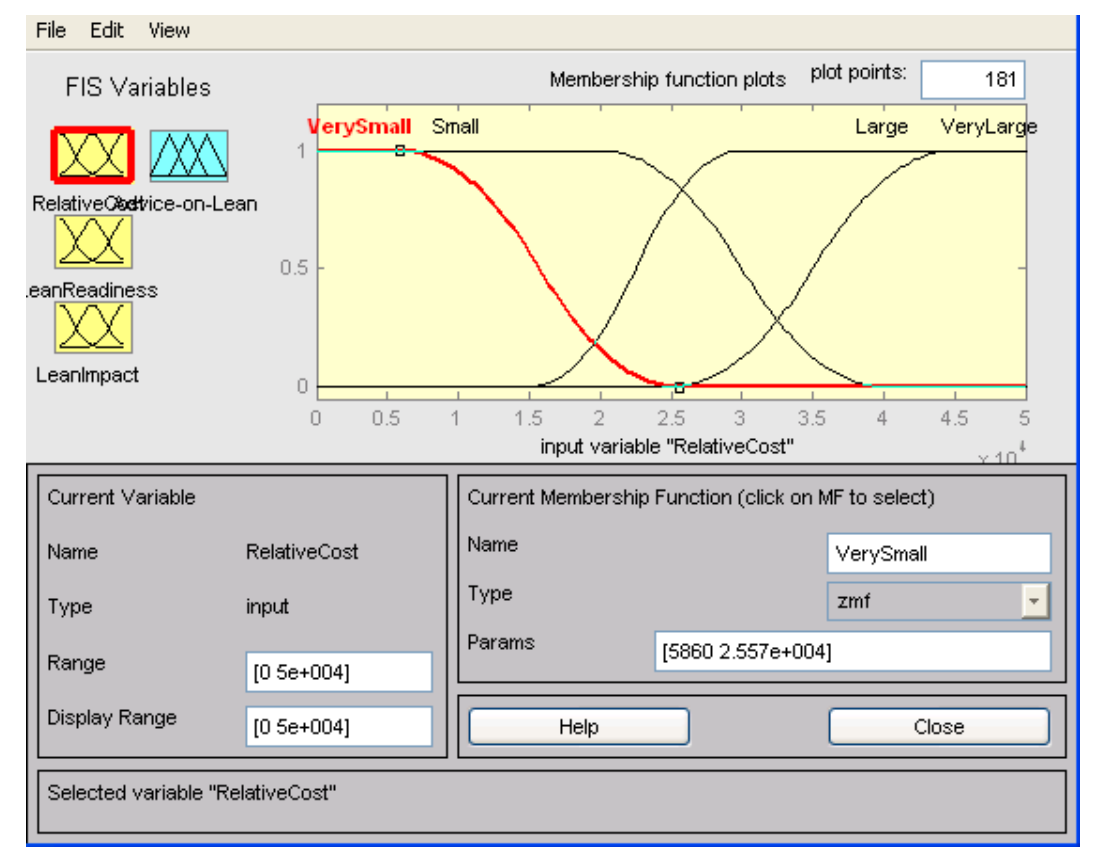

Figure 4: Illustration of the MFs of relative cost of the final system

Hence, if it is contended that the cost of lean implementation is VerySmall, the numerical representation implies that the cost value falls within a range of [0-25k]. Moreover, the interface allows the user to display and edit the ranges as desired. Furthermore, these functionalities are also typified in the membership functions of lean readiness and lean impact/benefit parameters.

However, the differentiations are on the choices of the linguistic variables adopted for each parameter. In this case, the lean readiness linguistic variables are defined chronologically as [NotReady, SomeWhatReady, JustAboutReady, Ready and Widespread]. The above-mentioned linguistic variables take the view that a NotReady posting would suggest that it would take a long time for a company to implement lean. 
Whereas, a Ready linguistic variable posting would suggest that a company in that category would less to implement lean.

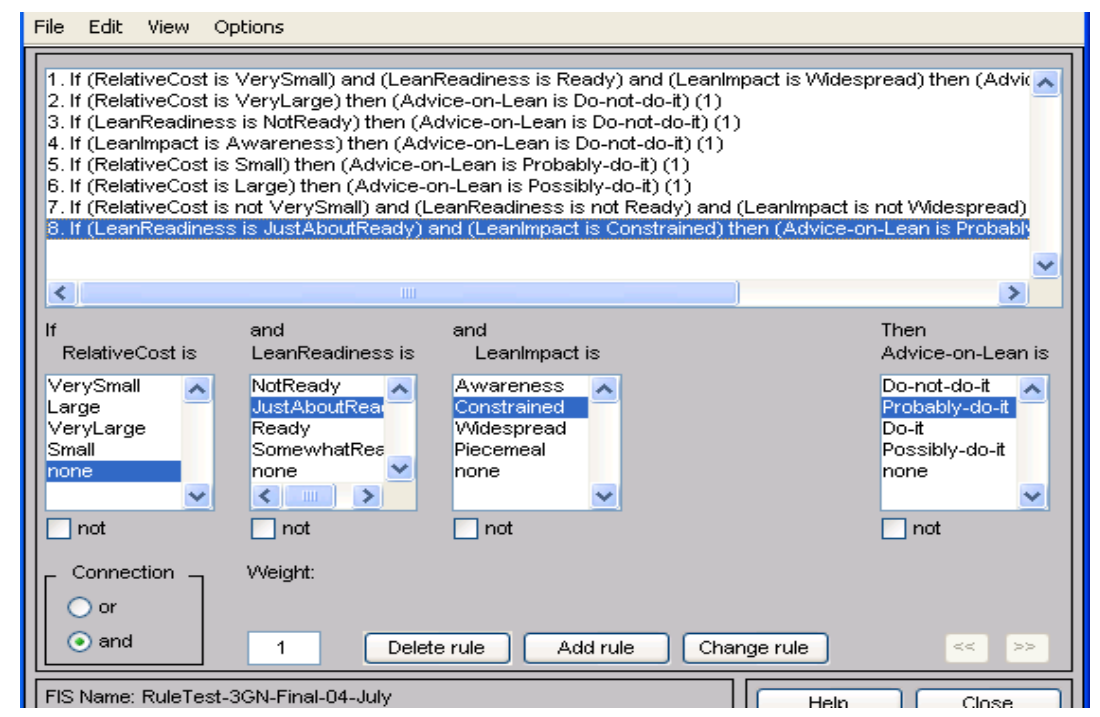

Figure 5: Interface of the rule editor

These variables represent the vector range of [1-10] of the elected parameter. On the benefit parameter, the FIS have involved the membership functions of inventory level, productivity level, cycle time and staff motivation. These membership functions have defined linguistic variables such as [Awareness, Piecemeal, and Constrained] 


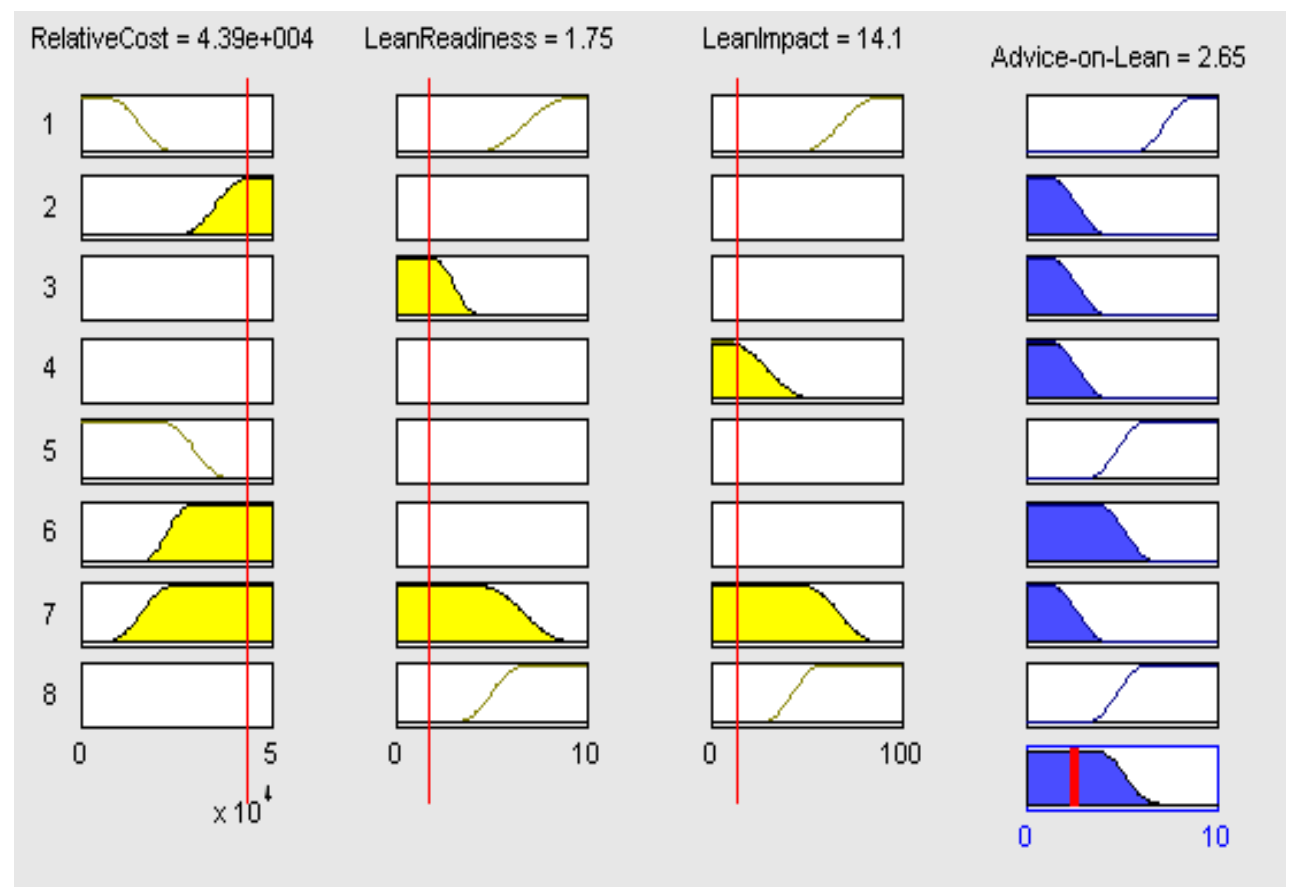

Figure 6: Rule viewer highlighting Negative points

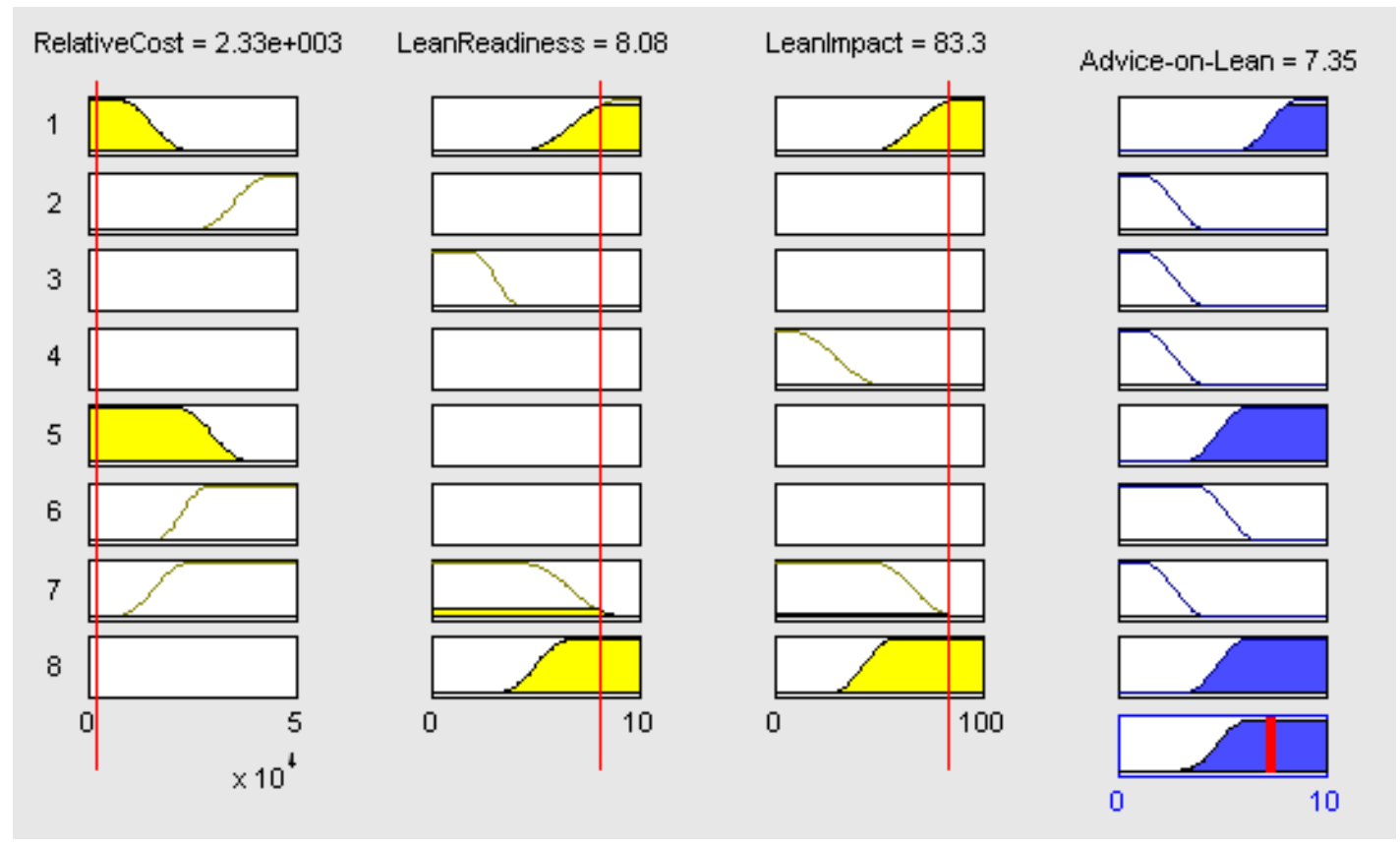

Figure 7: Rule viewer highlighting positive points

The system has generated eight final heuristic (HR) rules that are used for highlighting conditions for lean advice within SMEs as exemplified in Rule (4). 


\section{Rule 4: An example of the final heuristic rule in the main system}

HR: 1

If Relative-cost of lean is = VerySmall

$$
\begin{aligned}
& \text { and LeanReadiness }=\text { NotReady } \\
& \text { and LeanImpact }=\text { VeryLow } \\
& \text { then advice on lean }=\text { Do-not-do-it }
\end{aligned}
$$

\section{Assumption:}

The above rule takes into consideration the view that although the implementation cost of lean would be very small (between $0-5 \mathrm{k}$ ), company lean readiness does show a not ready status, meaning such a company has not implemented lean before, and has no trained personnel in lean perspectives. Additionally, the impact expected is very meagre. Therefore, based on these inferences, it is realistic for a company not to implement lean at all, at least for now.

\subsection{The Fuzzy-Logic Advisory System Outputs}

The final generated heuristic fuzzy-logic advisory rules highlighted in the previous section provide four different outputs which are the statements highlighting probable conditions for value advice to a potential lean user. These conditions were derived based on the analytical inferences and further consultative engagements with experts. It was believed having four statements that provide conditions of lean implementation present strong possible solutions in which a potential lean user may be guided as to whether he/she could implement lean manufacturing. More importantly, the documented four conditions were based on a simple language that may be easy to interpret by novice users. These statements are presented and discussed as follows.

\section{(i) Do it}

The model will return the advice statement 'do it' when all the indications are strongly and clearly in favour. The Lean Readiness rating will be high indicating that the company has invested in lean training, has staff with experience of implementing lean initiatives and has had success with previous initiatives. Lean impact will be moderate, to high indicating that there are expected to be financial and operational benefits of the proposed lean project. Cost of Implementation will be low indicating that there is little 
financial risk. Advice in this case would be simply to go ahead. This outcome might appear to be superfluous, a company in this position would hardly need to be told, but it is necessary to build confidence in the system. A user entering such parameters, even as a 'what-if' scenario, would expect to see a positive recommendation.

\section{(ii) Probably do it}

The model will return the advice statement 'probably do it' when there are strong indications in favour but also reason to pause for thought in at least one of the decision parameters.

- If Lean Readiness is high, then it is likely that reservations centre on the expected cost-benefit. It may be that costs seem relatively high, or lean impact seems relatively low. Advice in this case would be to reassess the costs and impacts and put in place risk management strategies to cover identified contingencies before proceeding.

- If Lean Readiness is low then this is the area to be addressed - because in this case the cost and impact parameters would necessarily be positive for the statement 'probably do it' to be returned. A number of steps could be taken to improve Lean Readiness, with further training, appointment of an internal lean champion, or an external lean consultant to supplement the company's own lean knowledge being the most obvious. Advice in this case would be contextdependent and focused on one or more of these actions.

\section{(iii) Possibly do it}

The model will return the advice statement 'possibly do it' when there are some positive indications but these are tempered by weaknesses in other parameters. If Lean Readiness is the strong indicator, then cost will be showing moderate-to-high and impact moderate-to-low. The danger in this case is that the company's lean capability and urge to improve will lead it into projects that do not return sufficient advantage for the business. 
- If cost can be reduced or the project revised to generate additional impact, then the recommendation could shift to 'probably do it', so advice would be to focus on these two points. There might still be a case for pursuing the project, even if it is marginal in cost-benefit terms, as a means to retaining and motivating lean resources in the company, but this should be a conscious decision.

- If Lean Impact is the strong indicator, then cost will be showing moderate-tohigh and readiness moderate-to-low. The danger in this case is that the company will pursue a project in the hope of high impact, but fail through high costs or over-ambition, and in doing so damage the prospects for future lean initiatives. Advice would be to break the project down into smaller steps, so that costs can be controlled and readiness improved by using each step as a training and leanawareness opportunity. Timescale will be increased but risks significantly reduced.

- If Cost is the strong indicator (i.e.: cost is very low), then impact and readiness will be showing moderate-to-low. The danger in this case is that the company might adopt the habit of a 'busy fool', pursuing small-scale projects that succeed individually but that collectively fail to generate sufficient impact. A case can be made for such a project if it is used deliberately to increase Lean Readiness, through training and experience, and the advice would be to proceed only if this is the case.

\section{(iv) Don't do it}

The model will return the advice statement 'don't do it' in the absence of a strong positive signal in any one of the parameters. The advice in this case would be to look for small-scale opportunities that start to improve the company's situation. A low cost, lowto-medium impact project that included training or knowledge-transfer through consultancy to improve Lean Readiness would be ideal. A series of such projects should see the company progressing until its proposals are rated 'possibly do it' then 'probably do it'. A company could also fine-tune the model to its own circumstances by adjusting the membership functions and rules, and then use it to test the comparative effect of a range of proposals. 


\section{System Applications and Validation}

Four case studies were conducted to determine the developed system's usability, relevance, accuracy and proof concept. This involved a number of experts both within academia and industry. Some of these included, Danfoss Randal Ltd, Hall Stage Ltd, Igranic Control Systems Ltd and PSJ Fabrications Ltd, all based in Bedfordshire, UK. Moreover, the experts engaged had good knowledge in the areas of engineering, lean manufacturing and soft computing.

\subsection{Expert inferences}

Validation sessions conducted with the four case studies yielded a number of inferences. In particular, the experts agreed on the assignment of numerical values into qualitative descriptions within the system. They upheld that usually, the cost of lean implementation within SMEs range from one thousand pounds as was the case in one of the external consultancy interventions, to anything up to $£ 50 \mathrm{k}$. SMEs are able to absorb these cost values provided they foresee immediate returns on the invested funds. Their inferences cite the current system development as a guiding path towards better impact assessment process using expert systems, a notion which is still at a very low level within these companies.

- They also concur on the readiness parameter as significant in offering advice on lean uptake. However, unlike relative cost and impact parameters that can easily be operationalised, the readiness parameter is hard to define let alone measure. Although they view the developed fuzzy-logic advisory system as a viable approach to lean impact assessment, the system should be simplified for eventual practical application in an industrial domain. Their key concern is the identification of the end user. This is due to the fact that the fuzzy-logic advisory system in their opinion seems to operate as a black-box, where SMEs, who are the intended users, may not have the technical expertise to make full use of it. They recommend that more work be carried out in simplifying the user interface and its applicability. However, they infer on the four statements seeking to advise a potential lean use [Do it, probably do it, possibly do and don't do it] respectively, as follows. The Validation session confirmed the researcher's 
earlier perception that someone would do it if they are ready with all the required resources, and foresee realistic returns. [Do not do it] statements shall imply a company has not reached the benchmarking of implementing lean due to its lack of resources and other necessary ingredients such as awareness. However, in their view, such statements are linear logic given elevated prominence by the results from the fuzzy-logic advisory system. Therefore, future work requires the deployment of a consultant or expert system in order to interpret the results for the end user.

- The experts' inferences on the behavioural patterns of the surface viewers in Figures (8) and (9) run as follows.

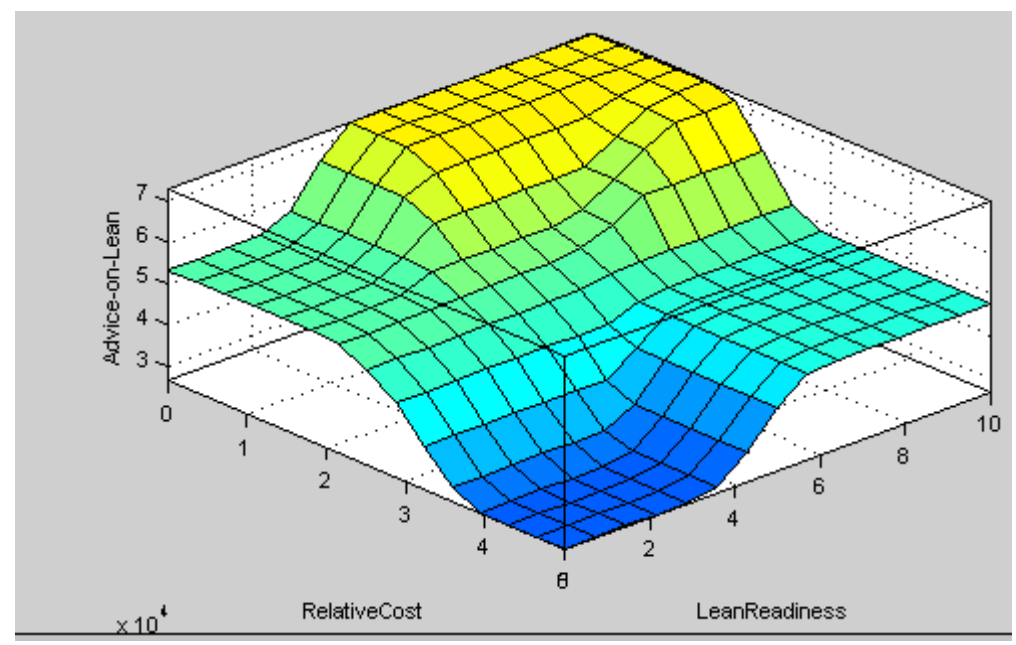

Figure 8: Topography of the relative cost v. lean readiness

The apex portion of the topography of Figure (8) demonstrates a scenario where a company's position in terms of lean advice is to do it straight away. This is due to the fact that at the orange lining, the relative cost is really low yet the company lean readiness is extremely high. In a scenario where cost begins to climb higher and the company lean readiness is interpreted as not very high, hence a degree of probability of lean adoption may ensue. This continues with the incremental rise in relative cost and decline in the lean readiness status where lean advice would ultimately become untenable (do not do it). 


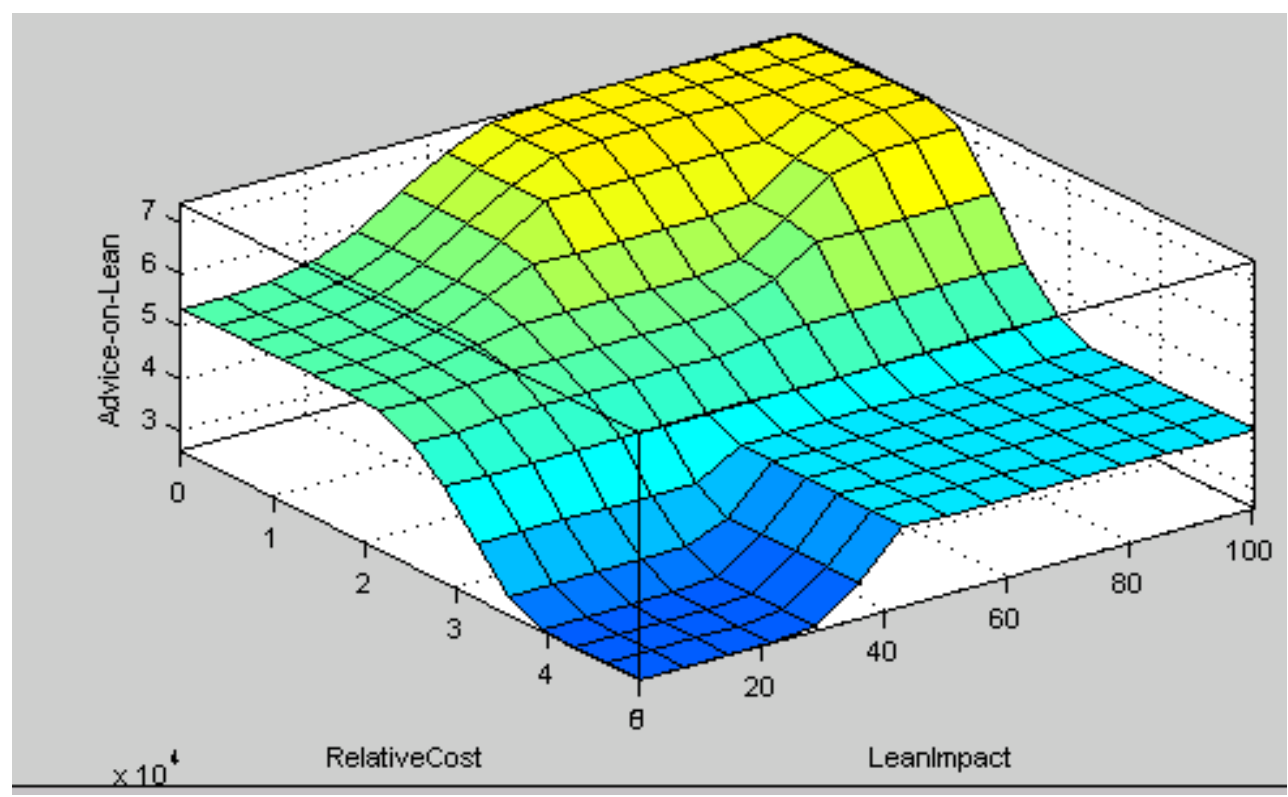

Figure 8: Topography of relative cost v. lean impact

- In Figure (9), a similar reasoning was adopted on the relative cost of lean manufacturing implementation; save for the related variable that in this instance became the lean impacts (expected benefits).

The experts were of the opinion that if relative cost is low and the forecast lean impact is high, companies would be urged do it straight away. Conversely, if the expected impact is anticipated to be lower than the cost of implementation and the cost is unrealistically high, the advice would be not to do it. The interpretive view on Figure (10) by the experts was that the steep drop from the orange portion of doing it to greenish parts (possibly doing it) may be ascribed to a number of reasons. For example, an individual company may have a strategy of deploying lean manufacturing such as having a change agent to champion the cause which is why a decision may be sudden and not gradual in this context as illustrated in the topographic view of the surface. 


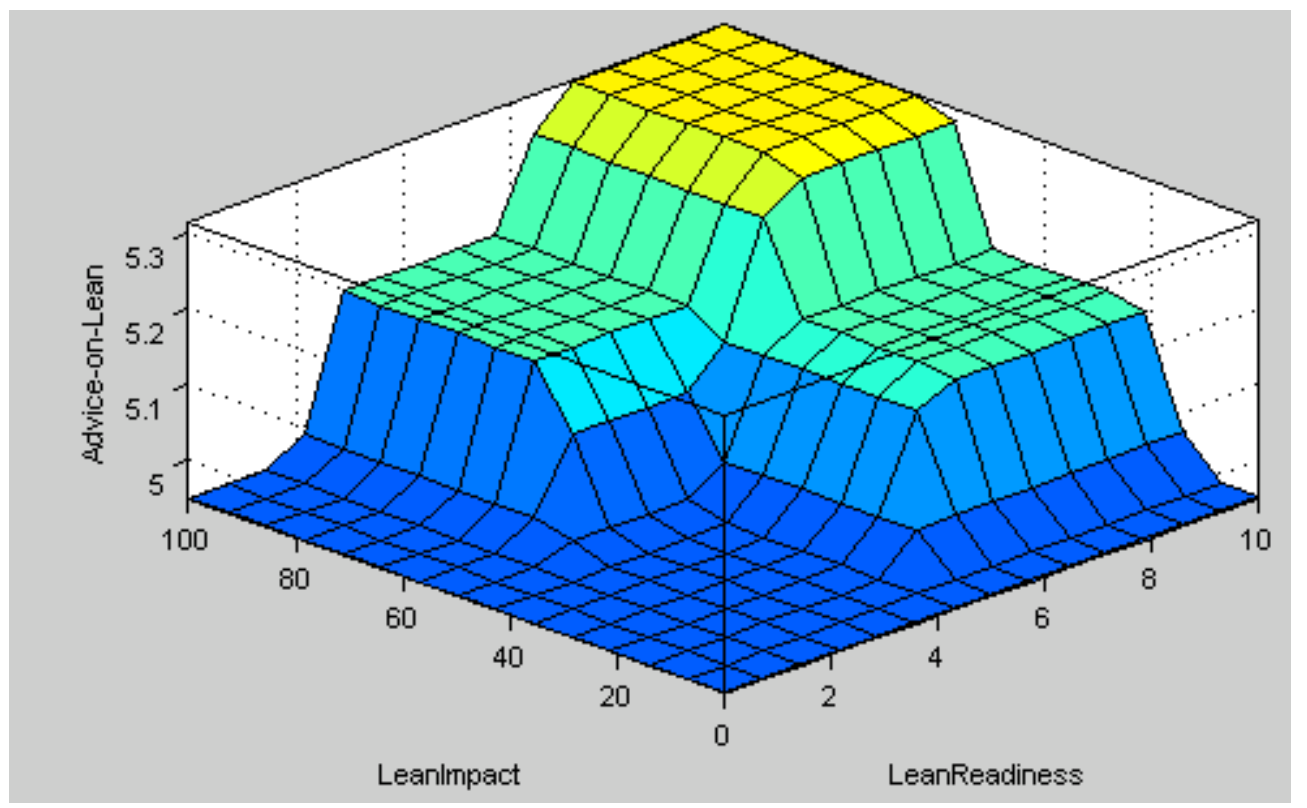

Figure 10: Topography of the lean impact v. lean readiness

Never-the-less, the experts acknowledged that the system has the capability of providing an overview of the parameters (relative cost, readiness and impact). Moreover, they believe the approach of using a fuzzy-logic advisory system to assess the lean impact is novel and provides a sound logic in giving advice on whether to implement the concept or not, based on the impact assessment scenarios. In particular, they like the fuzzy-logic advisory system surface curves that highlight relationships between the listed parameters in a systematic manner.

Illustratively, it can be seen from the surface viewer highlighted in Figure (8) that if relative cost of implementing lean manufacturing decreases or remains very small and the other parameters (readiness and impact) are higher, there is an inclination of a business desire to implement lean manufacturing (do it). Conversely, if the cost was zero, and the other impacts are higher, a business may not probably do it because of the perception that cheap things might not have value. However, the curve tends to highlight that as relative cost begins to increase gradually with readiness and impacts, there is a higher inclination of making a decision to "do it", because the cost is not zero and not very high, yet realistically acceptable in comparison to the level of impacts due to be achieved thereafter. In terms of the of input parameters such as employees skills, the experts views are that employees' skills should be examined critically, because 
employees' skills alone as a parameter cannot be used on its own to interpret the topographical representation. This is because employees' skills can be readjusted provided management can accept and allow or give them the opportunity to perform without restrictions or blame. In the experts' opinions, evaluation of management support should be treated on what they (managers) do and not what they say, as quite often the inverse is true.

Additionally, the experts further concurred that, quite often there is a tendency of a lack of understanding in the impact assessment scenario, as people tend to overstate benefits and yet underestimate costs because it makes things (their business) appear better. Therefore understanding the overall business structure is crucial. Business should look at the start and end of their supply-chain so they can order the required supplies, manufacture products making the order quantity and supply the customer in accordance within their capability. The experts conclusively agreed with the heuristic rules embedded within the fuzzy-logic advisory system and maintain that they give meaningful inferences, of choices of advice to take while making a decision based on the fuzzy-logic advisory system guidance.

For example, the rule matrix in Figure (7) provides an indicator that if cost is very low and the company readiness is very low and the expected impact indicator is very low as well, one may be inclined into absorbing it. The essence of this thinking is based on the premise that if relative cost is very low, companies may assume that by bringing in lean, they could do something on their readiness perspective and impact factor as a means of improving their positions.

The experts' opinion on the fuzzyfication of the linguistic variables was also unanimous. They agreed on the fuzzy-logic advisory system input linguistic variables and the resultant outputs of; Don't Do It, Possibly Do It, Probably Do It and Do It respectively. However, as demonstrated in Table (2), the lean experts inferred that by combining the choices of levels of the relative cost, readiness and impact parameters, one could make an informed decision based on the pieces of advice provided herein. The rule viewer seems to back the designed rule logic that implies lean should not be 
implemented (Do not do-it) in such a scenario where there is absence of a strong positive signal in any one of the parameters highlighted above.

Table 2: A comparison of the experts' score versus the fuzzy-logic advisory system'

\begin{tabular}{|c|c|c|c|c|}
\hline 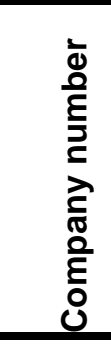 & 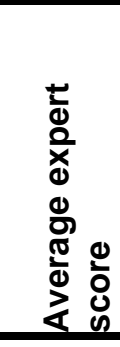 & 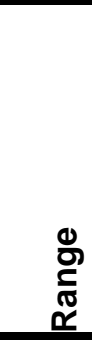 & 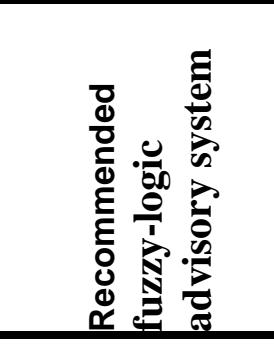 & 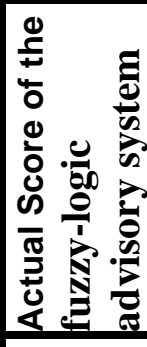 \\
\hline 1.0 & 83.5 & 30.0 & Do It & 90.0 \\
\hline 2.0 & 75.0 & 40.0 & Possibly Do It & 65.0 \\
\hline 3.0 & 62.0 & 25.0 & Possibly Do It & 65.0 \\
\hline 4.0 & 8.0 & 20.0 & Don't It & 45.0 \\
\hline 5.0 & 72.5 & 20.0 & Probably Do It & 70.0 \\
\hline 6.0 & 93.5 & 15.0 & Do It & 90.0 \\
\hline 7.0 & 80.0 & 10.0 & Do It & 90.0 \\
\hline 8.0 & 71.5 & 20.0 & Probably Do It & 65.0 \\
\hline 9.0 & 60.0 & 20.0 & Possibly Do It & 65.0 \\
\hline 10.0 & 14.0 & 30.0 & Don't It & 45.0 \\
\hline
\end{tabular}

This is because; the cost is perceived as enormous, and the company is returned as not ready at all. Additionally, the expected returns on the lean investment are postulated as meagre. Therefore, the experts agreed with the validity of the operationalisation of the developed fussy system, as in their opinion, it provides realistic, logical and relevant guidance for decision-making processes on lean uptake.

\section{Conclusions}

The development of a fuzzy-logic advisory system for lean manufacturing within SMEs has been described. The research study has provided a novel system for assessing the impact of lean manufacturing within SMEs. This system is intended to aid SME practitioners in predicting the impact of lean manufacturing implementation at the early implementation stage. Moreover, companies can also use the system to assess their eligibility in terms of lean readiness. This is important because the degree of a company lean readiness determines whether it does succeed in implementing lean. The level of 
readiness may also determine the amount of resource needs for the lean project. The contribution will therefore be beneficial to business community in several ways as listed below.

- It can enable an organisation to forecast the probable relative cost of implementing lean manufacturing within its business.

- A company can also project upfront what it anticipates to achieve as the return on investments (ROI) from implementing lean manufacturing.

- The framework is a precursor for implementing the concept of lean manufacturing in a business. This is because it allows a potential lean using company to make assessments on its capabilities and the capacity of its resources for the intended project.

- Organisations are able to realise their degree of lean need, since the framework conducts test-case scenarios of the affected business drivers as a qualification for lean embracement.

- Identification of area of need is conducted by carrying out analysis on factors such as resource availability vis-à-vis the extent of the problem to be solved.

- Companies can also evaluate the strengths and weaknesses of their manufacturing processes based on the impact assessment results.

- The framework can also be used as a standard business tool for assessing an organisation's performance status.

- Future work should seek to refine the developed system's interface with easy-touse features, which has the capability of pinpointing the different areas identified within the topography.

\section{Acknowledgements}

The authors would like to thank the Engineering Physical Science Research Council (EPSRC), the Manufacturing Advisory Service in the East of England (MAS-East) and Cranfield University, for sponsoring this research project.

\section{References}


- Achanga, P., (2007), Development of an impact assessment framework for lean manufacturing within SMEs, PhD thesis, Cranfield University, UK.

- Achanga, P., Shehab, E., Roy, R. and Nelder G. (2006a), "Lean impact assessment at the conceptual design stage", Proc. of the $16^{\text {th }}$ International Design Seminar (CIRP 2006), CIRP Publishers, Kananaskis, Alberta, Canada.

- Achanga, P., Shehab, E., Roy, R., and Nelder, G. (2006b), Critical success factors for lean implementation within SMEs, Journal of Manufacturing Technology Management, Vol: 17 No.4. pp.460-71.

- Achanga, P., Shehab, E., Roy, R., and Nelder, G. (2005), Lean Manufacturing for SMEs: enabling rapid response to demand changes, Proceedings of the $15^{\text {th }}$ International Conference on Engineering Design (ICED 2005), Institution of Engineers, Melbourne, Australia, August 2005, ISBN: 1-904670-00-8, pp.148.

- Agyapong-Kodua, K., Ajaefobi J.O., and Weston, R.H., (2009), Modelling dynamic value streams in support of process design and evaluation, International Journal of Computer Integrated Manufacturing, Vol. 22, No. 5, May 2009, 411-427.

- Collantes, L., Roy, R. and Madill, J. (1999), Fuzzy process evaluation using a fuzzy expert system, RASC'99, Springer-Verlag, London.

- Cook, C.R and Graser, J.C. (2002), The effects of lean manufacturing, Rand Publishers, Santa Monica CA, ISBN 083303023.

- Denton, P.D. and Hodgson, A. (1997), Implementing strategy-led BPR in a small manufacturing company, Fifth International Conference on FACTORY 2000, UK - The Technology Exploitation Process Conference Publication No.435, p. 1-8.

- Hicks, B.J., and Matthews, J. (2010), The barriers to realising sustainable process improvement: a root cause analysis of paradigms for manufacturing systems improvement, International Journal of Computer Integrated Manufacturing, Vol. 23, No. 7, July 2010, 585-602.

- Hines, P., Howleg, M. and Rich, N. (2004), Learning to evolve, a review of contemporary lean thinking, International Journal of Operations and Production Management, Vol.24 No.10, pp.994-1011.

- Kulak, O., Durmuşoğlu, M.B. and Kahrmaman, C. (2005), Multi-attribute equipment selection based on information axiom, Journal of Materials Processing Technology Vol: 169 p. 337-345. 
- Lau, H.C.W., Ning, A., Pun, K.F., Hin, K.S. and Ip, W.H. (2005), A knowledge-based system to support procurement decision, Journal of Knowledge Management, Vol.9 No.1, pp.87-100.

- Levy, J. (1993), Medium sized enterprises-a recipe for success, Manufacturing Engineer, IEE, Vol.72 No.6, pp.258-9.

- Liang, G.S. and Wang, M.J. (1991), A fuzzy-multicriterion decision making method for facility site location, International Journal of Production Research, Vol: 29 No.11 p.2313-2330.

- Muthu, S., Devadasan, S.R, Mendonca, P.S. and Sundararaj, G. (2001), Preauditing through a knowledge base system for successful implementation of a QS 9000 based maintenance quality system, Journal of Quality Management, Vol.7 No.2, pp.90-103.

- Murman, E., Allen, T., Bozdogan, K. (2002), Lean enterprise value, Insights from MIT's Lean Aerospace Initiative, Palgrave Publishers, New York: NY, ISBN 0333976975.

- Northrup, C.L. (2005), Bridging the gap to lean accounting, the Northwest Lean Networks, http://www.nwlean.net, (accessed on $23^{\text {rd }}$ May 2007).

- Panizzolo, R. (1998), Applying the lessons learned from 27 lean manufacturersthe relevance of relationships management, International Journal of Production Economics, Vol.55, pp.43-55.

- Parent, R., Roy, M., and St-Jacques, D. (2007), A system-based dynamic knowledge transfer capacity model, Journal of Knowledge Management, Vol.11 No.6, pp.81-93.

- Rao, A.V.S and Pratihar, D.K. (2006), Fuzzy logic-based expert system to predict result of infinite element analysis, Knowledge Based Systems, Vol.20 No.1, pp.37-50.

- Reid, R.A. and Koljonen E.L. (1999), Validating a manufacturing paradigm: a system dynamics modelling approach Proceedings of the 1999 Winter Simulation Conference, 5 - 8 December 1999, Phoenix, Az, U.S.A., pp 759-765

- Roy, R. (2003), Cost Engineering: Why, What and How? Decision Engineering Report Series, No 1, Cranfield University.

- Stanworth, J. and Purdy, D. (2003), SME fact and issues, a compilation of current data and issues on UK small and medium sized firms, Labour Financial and Industry Group (LFIG), Westminster Business School. 
- Shah, R. and Ward, P.T. (2003), Lean manufacturing: context, practice bundles and performance, Journal of Operations Management, Vol.21, pp.129149.

- Simmons, L., Holt, R,. Dennis, D., Walden, C., (2010), Lean Implementation in a low volume manufacturing environment: a case study, Proceedings of the 2010 Industrial Engineering Research Conference, A. Johnson and J. Miller, eds., Mississippi State University - Centre for Advanced Vehicular Systems Extension Canton, MS 39046, USA.

- Shehab, E.M. and Abdalla, H.S. (2002), A design to cost system for innovative product development, Proc. of Institution of Mechanical Engineers, Journal of Engineering Manufacture, Vol.216 Part B, PP.999-1019.

- Singh, R.K., Kumar, S., Choudhury, A.K., and Tiwari, M.K. (2006). "Lean tool selection in a die casting unit: a fuzzy-based decision support heuristic." International Journal of Production Research, Vol: 44No. 7 p.1399-1429.

- Zadeh, L. (1975), "The concept of a linguistic variable and its application to approximate reasoning-1", Information Sciences, Vol: 8, 199-249.

- Zulfiqar, K. and Rajeev, B. (2007), Identifying the need for world class manufacturing and best practice for SMEs in the UK, International Journal of Management and Enterprise Development, Vol.4 No.4, pp.428-440. 